\title{
Efficient and Robust Kerr-Lens Mode-Locking of Cr:LiSAF Lasers Using Gain-Matched Output Couplers
}

\author{
Ferda Canbaz ${ }^{1}$, Ersen Beyatli ${ }^{1}$, Li-Jin Chen ${ }^{2}$, Alphan Sennaroglu ${ }^{1}$, Franz X. Kärtner ${ }^{2,3}$, and Umit Demirbas ${ }^{4, *}$ \\ ${ }^{1}$ Laser Research Laboratory, Departments of Physics and Electrical-Electronics Engineering, Koç University, Rumelifeneri, Istanbul, Turkey \\ ${ }^{2}$ Department of Electrical Engineering and Computer Science, Massachusetts Institute of Technology, Cambridge, Massachusetts 02 139, USA \\ ${ }^{3}$ Center for Free-Electron Laser Science, DESY and Department of Physics, University of Hamburg, D-22607 Hamburg, Germany \\ ${ }^{4}$ Laser Technology Laboratory, Department of Electrical and Electronics Engineering, Antalya International University, Antalya, Turkey \\ *Corresponding Author: umit79@alum.mit.edu
}

\begin{abstract}
We have demonstrated robust and efficient Kerr-lens mode-locked operation of a lowcost, diode-pumped Cr:LiSAF laser using a gain-matched output coupler. Sub-30-fs pulses with 80 -pJ pulse energy have been demonstrated with $80 \mathrm{~mW}$ of pump power.

OCIS codes: (320.7090) Ultrafast lasers; (140.4050) Mode-locked lasers; (310.6845) Thin film devices and applications.
\end{abstract}

\section{Introduction}

Kerr-lens mode-locking (KLM) is a very well-known and versatile technique that is widely used for the generation of ultrashort pulses from broadband gain media such as Ti:Sapphire [1, 2], Cr:ZnSe [3], Cr:Forsterite, and Cr:LiSAF [4-8]. KLM generates an artificial saturable absorber with an instantaneous lensing effect that shortens the circulating intracavity pulsewidth via self-amplitude modulation (SAM). Hence, unlike saturable Bragg reflectors, KLM does not impose any bandwidth limitation effect and the obtainable pulsewidth is limited only by the gain bandwidth of the lasing material and dispersion bandwidth of the laser cavity. To achieve the shortest pulses: (i) flat dispersion profile over the whole gain bandwidth should be utilized, and (ii) strong gain filtering effect of the active medium should be overcome. To fulfill the second requirement, a large SAM is desired, which necessitates the use of low output coupling, high pump powers, critical cavity alignment, and, in some cases, operation near the edge of the stability range. This may result in reduced output beam quality, lower laser efficiency, high mode-locking threshold, and stability problems in long-term operation. These issues are even more significant in gain media with a low nonlineer refractive index $\left(n_{2}\right)$ such as Cr:LiSAF $\left(n_{2}=0.8 \times 10^{-16} \mathrm{~cm}^{2} / \mathrm{W}\right.$, four times lower than that for Ti:Sapphire [9]). Even though pulses as short as 10 fs have been demonstrated from low-cost diode-pumped Cr:LiSAF lasers [4], the ultrafast technology based on KLM Cr:LiSAF lasers could not be developed beyond laboratory demonstrations due to the above mentioned problems,.

To overcome the limitations imposed by KLM, the gain filtering effect should be minimized. In that respect, Chen et al. recently suggested the use of gain matched output couplers (GMOC) with a transmission profile that matches the gain spectrum of the laser active medium[10]. This creates a loss profile that has the same shape as the cavity gain, which results in a broadband flat net gain profile. Then, this eliminates the need for strong SAM, and a low level of KLM action is sufficient to sustain ultrashort pulses. By employing a 4\% GMOC in a Ti:Sapphire laser, Chen et al. demonstrated robust mode-locking at greatly-reduced Kerr nonlinearity requirements [10], and the laser produced sub-8-fs pulses with excellent beam quality.

In this study, we employed a gain matched output coupler to demonstrate robust KLM operation of a diodepumped, low-cost Cr:LiSAF laser with long-term stability. The laser is pumped with a $130 \mathrm{~mW}$ diode having a diffraction-limited output beam at the wavelength of around $660 \mathrm{~nm}$. KLM operation did not require careful cavity alignment and could be initiated at incident pump powers as low as $50 \mathrm{~mW}$. Once initiated, the system could stay in KLM operation for hours. At an incident pump power of only $80 \mathrm{~mW}$, the Cr:LiSAF laser produced almost transform limited sub-30-fs pulses around $850 \mathrm{~nm}$ at a repetition rate of $120 \mathrm{MHz}$. The average output powers were around $10 \mathrm{~mW}$ and the corresponding peak powers reached $4 \mathrm{~kW}$ level. Moreover, the system had an estimated material cost below $\$ 5 \mathrm{k}$, an electrical-to-optical conversion efficiency of around 5\%, a footprint of only $30 \mathrm{~cm} \times 40$ $\mathrm{cm}$ and could be powered with 8 AA-type batteries for 10 hours [11]. The obtained pulsewidth was limited by the dispersion bandwidth of the available mirror set, and sub-10-fs pulses should be possible in future by using a dispersion optimized mirror set. We believe that compact, low-cost and efficient KLM Cr:LiSAF lasers might become an attractive alternative to the existing Ti:Sapphire laser systems in various areas of science and technology.

\section{Experimental Setup}

Figure 1 (left) shows a schematic of the KLM Cr:LiSAF laser. The pump diode beam was first collimated with a 4.5 $\mathrm{mm}$ focal length aspheric lens (f1) and then focused into the crystal using a $60 \mathrm{~mm}$ focal length achromatic doublet (f2). The Cr:LiSAF crystal was 1.5\% Cr-doped, had a length of $6 \mathrm{~mm}$ and absorbed around $98 \%$ of the incident 
pump power. The laser cavity consisted of two curved mirrors, each with a radius of curvature of $75 \mathrm{~mm}(\mathrm{M} 1, \mathrm{M} 2)$, three flat high reflectors (M3-M5), and the GMOC mirror. The GMOC had a transmission of around $0.7 \%$ around the gain peak and its spectral transmission profile matched the gain profile of Cr:LiSAF quite well [Fig. 1 (right)]. Dispersion compensation has been employed using commercial double-chirped mirrors (DCMs, M3-M5), which provided around $-50 \mathrm{fs}^{2}$ of GDD per bounce. The total cavity dispersion was estimated to be around - $50 \mathrm{fs}^{2}$, but was not as broadband as desired due to the limited bandwidth of the available commercial DCM mirrors [Fig. 2 (right)].
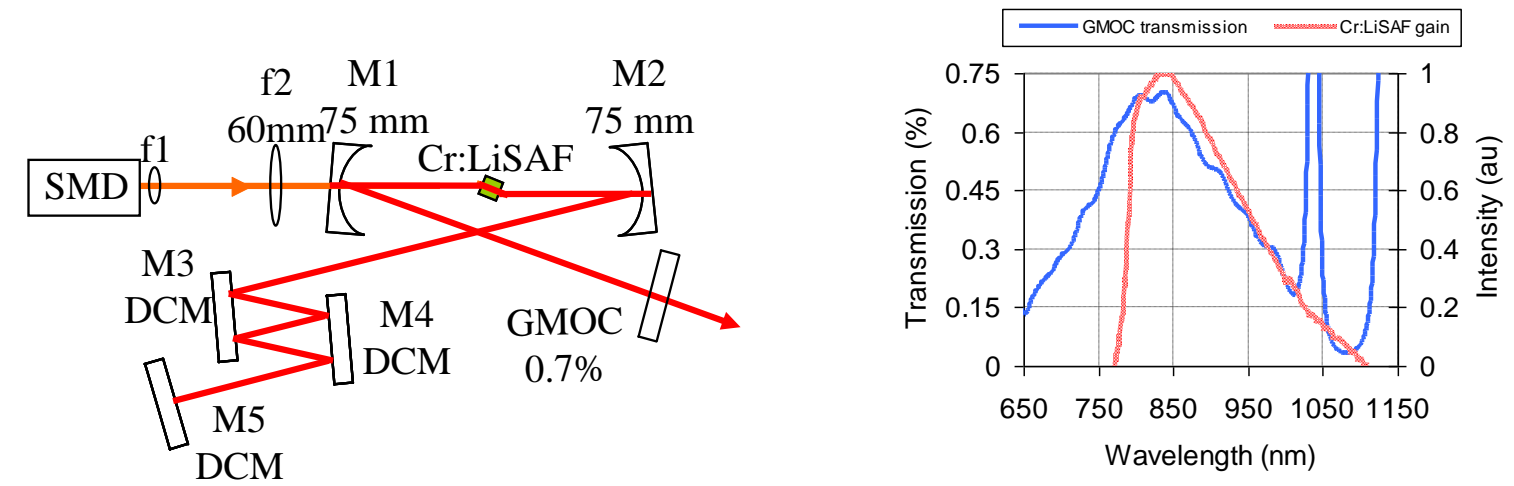

Fig. 1. (Left): Schematic of the single-mode diode (SMD) pumped Kerr-lens mode-locked Cr:LiSAF laser with a gain-matched output coupler (GMOC). (Right) Gain profile of the Cr:LiSAF crystal and the designed transmission spectrum of the GMOC .

\section{Results and Discussion}

Fig. 2 (left) shows the measured variation of the Cr:LiSAF laser output power with incident pump power. The cw lasing threshold was around $20 \mathrm{~mW}$, and the laser operated in only pure $\mathrm{cw}$ regime for pump powers up to around $40 \mathrm{~mW}$. The slope efficiency in the $\mathrm{cw}$ regime was around $20 \%$. For pump powers above $50 \mathrm{~mW}$, it was possible to initiate mode-locked operation by slightly shaking one of the cavity end mirrors. Once mode-locked, the laser output beam had a symmetric beam profile and operated in the TEM $_{00}$ mode [Fig. 2 (middle)]. The variation of the optical spectrum of the pulses with input pump power is shown in Fig. 1 (right). We have obtained spectral widths as large as $50 \mathrm{~nm}$ around $850 \mathrm{~nm}$ at the full pump power of $135 \mathrm{~mW}$. This available bandwidth has the potential to generate as short as 15 -fs pulses assuming a transform limited $\mathrm{sech}^{2}$ temporal profile. Figure 2 (left) also shows the estimated variation of the total cavity dispersion in the spectral region of interest. We believe that the sharp variation of GDD above $920 \mathrm{~nm}$ limited the obtainable spectral width in this study. We have also noticed that, for pump powers above $100 \mathrm{~mW}$, the output pulses were chirped and it was not possible to obtain transform limited pulses via simple dispersion compensation outside the cavity (using DCM mirrors or prism pairs). We believe that variations in the GDD in the 880-920 nm range might be causing this excess chirp as the spectra extend into this region at pump powers above $100 \mathrm{~mW}$. For example, at the pump power of $135 \mathrm{~mW}$, the measured pulsewidth is $27 \mathrm{fs}$ long, corresponding to a time-bandwidth product of 0.57 . The average output power was $15 \mathrm{~mW}$, corresponding to pulse energy of $125 \mathrm{pJ}$ and a peak power of $4.1 \mathrm{~kW}$ at a repletion rate of $120 \mathrm{MHz}$.
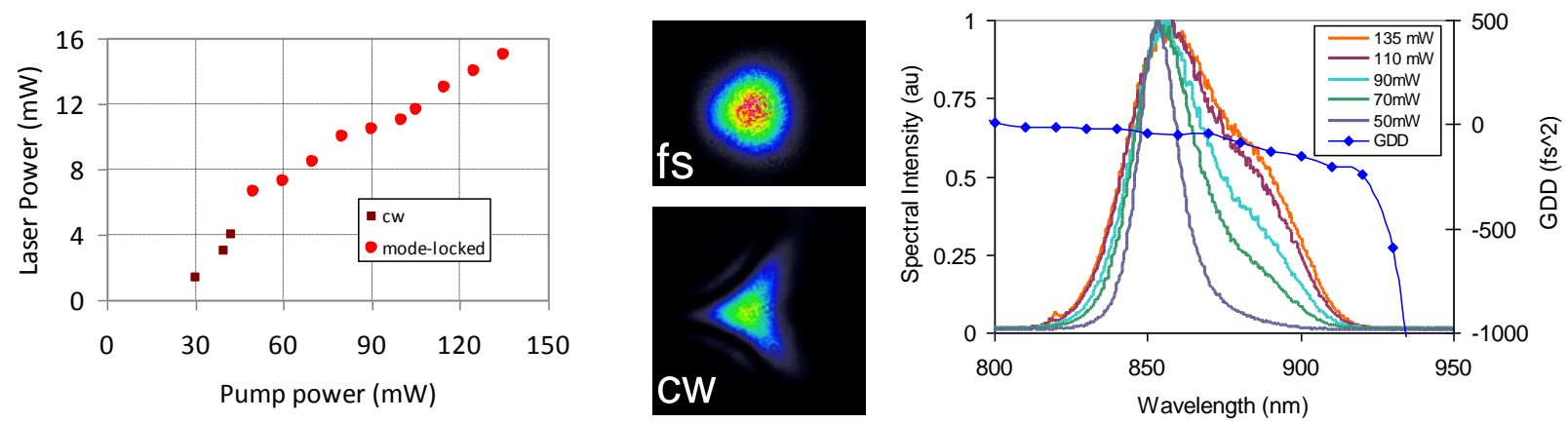

Fig. 2. (Left) Measured variation of the Cr:LiSAF laser output power as a function of incident pump power. (Middle) Measured output beam profile for mode-locked (fs) and continuous-wave cases. (Right) Measured variation of the laser output spectrum with incident pump power.

On the other hand, for pump powers below $100 \mathrm{~mW}$, the obtained pulses were chirp free. As an example, Fig. 3 below shows the measured pulse train characteristics at an incident pump power of $80 \mathrm{~mW}$. For this case, the optical spectrum was centered around $860 \mathrm{~nm}$ and had a FWHM of $28 \mathrm{~nm}$ [Fig 3 (left)]. The measured pulsewidth with interferometric autocorrelation is 29 femtosecond long (assuming $\operatorname{sech}^{2}$ pulse shape) and the corresponding time 
bandwidth product is 0.331 [Fig 3 (middle)]. The repetition rate was around $119.5 \mathrm{MHz}$ and the measured radio frequency (RF) spectrum of the pulse train showed a clean RF peak without any noise sidebands [Fig 3 (right)]. The mode-locked average output power was $10.2 \mathrm{~mW}$, and the corresponding pulse energy and peak powers were $84 \mathrm{pJ}$ and $4 \mathrm{~kW}$, respectively.
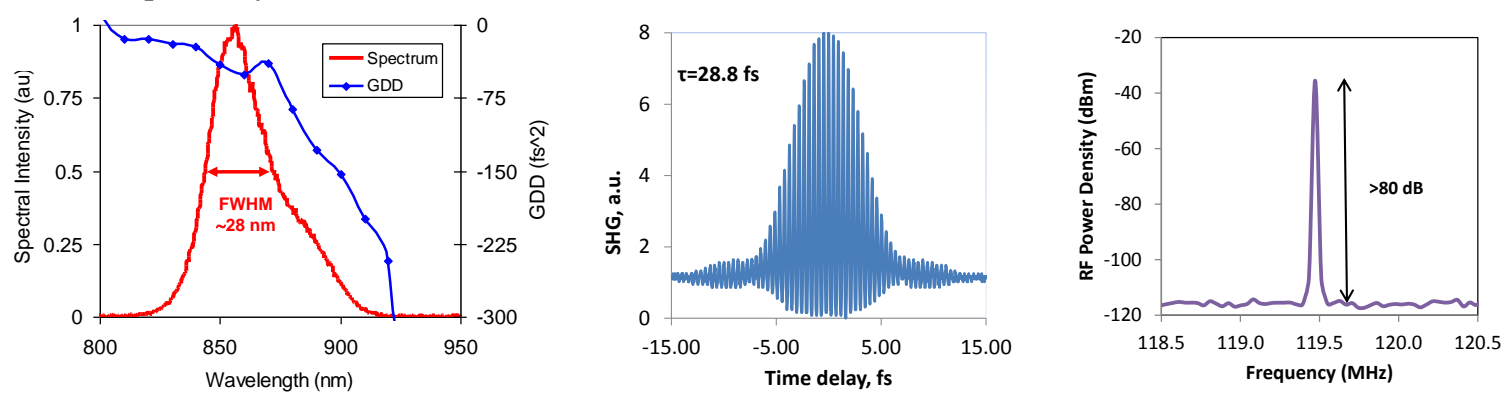

Figure 3: (Left): Measured optical spectrum of the femtosecond Cr:LiSAF laser taken at an incident pump power of $80 \mathrm{~mW}$. The spectrum had a bandwidth of $28 \mathrm{~nm}$ centered around $860 \mathrm{~nm}$. Calculated group delay dispersion profile of the cavity is also shown. (Middle) corresponding interferometric autocorrelation trace of the femtosecond pulses at $80 \mathrm{~mW}$ incident pump power. (Right) Measured radio frequency spectrum of the pulse train.

We note here that, from the same system, we could not obtain stable KLM operation by using regular output couplers. Hence, our study clearly demonstrates that gain-matched output coupler technology enables robust KLM operation with long-term stability even in laser gain media with very low nonlinear refractive index. Moreover, with the GMOC, we did not need to operate the Cr:LiSAF laser near the edge of the stability region to obtain KLM and this enabled higher average output powers with good beam quality. For example, we could reach an optical-tooptical conversion efficiency of $12.5 \%$ in our study, which is in a very good position compared to earlier KLM Cr:LiSAF results in literature (10 fs pulses with $0.27 \%$ efficiency in [4], 12 fs pulses with $0.76 \%$ efficiency in [5], 26 fs pulses with $0.78 \%$ efficiency in [6], 42 fs pulses with $5.25 \%$ efficiency in [7], 80 fs pulses with $11 \%$ efficiency in [8]).

\section{Conclusions}

In conclusion, we have employed a gain-matched output coupler to demonstrate robust KLM operation of a diodepumped, low-cost Cr:LiSAF laser with long-term stability. An electrical-to-optical conversion efficiency of around 5\% have been attained, which is around two orders of magnitude better than what is achieved with Ti:Sapphire systems. Obtained pulsewidths (sub-30-fs) were limited by the dispersion profile of the available DCM mirror set, and sub-10-fs pulses should be possible with careful dispersion compensation. We believe that, with future progress, low-cost, compact and efficient KLM Cr:LiSAF lasers with GMOC technology have the potential to be an attractive laser source for many scientific and technological applications.

\section{References}

[1] D. E. Spence, Kean, P. N., Sibbett, W., "60-fsec pulse generation from a self-mode-locked Ti:sapphire laser," Optics Letters, vol. 16, 1991.

[2] R. Ell, U. Morgner, F. X. Kärtner, J. G. Fujimoto, E. P. Ippen, V. Scheuer, G. Angelow, and T. Tschudi, "Generation of 5 fs pulses and octave-spanning spectra directly from a Ti:sapphire laser," Optics Letters, vol. 26, pp. 373-375, 2001.

[3] M. N. Cizmeciyan, H. Cankaya, A. Kurt, and A. Sennaroglu, "Kerr-lens mode-locked femtosecond Cr2+:ZnSe laser at $2420 \mathrm{~nm}$," Optics Letters, vol. 34, pp. 3056-3058, Oct 152009.

[4] S. Uemura and K. Torizuka, "Generation of $10 \mathrm{fs}$ pulses from a diode-pumped Kerr-lens mode-locked Cr : LiSAF laser," Japanese Journal of Applied Physics Part 1-Regular Papers Short Notes \& Review Papers, vol. 39, pp. 3472-3473, Jun 2000.

[5] S. Uemeura and K. Torizuka, "Generation of 12-fs pulses from a diode-pumped Kerr-lens mode-locked Cr:LiSAF laser," Optics Letters, vol. 24, pp. 780-782, 1999

[6] S. Uemura and K. Miyazaki, "Femtosecond Cr:LiSAF laser pumped by a single diode laser," Optics Communications, vol. 138, pp. 330332, 1997.

[7] M. J. P. Dymott and A. I. Ferguson, "Self-mode-locked diode-pumped Cr:LiSAF laser producing 34-fs pulses at 42-mW average power," Optics Letters, vol. 20, pp. 1157-1159, 1995.

[8] A. Robertson, R. Knappe, and R. Wallenstein, "Diode-pumped broadly tunable (809-910 nm) femtosecond Cr : LiSAF laser," Optics Communications, vol. 147, pp. 294-298, Feb 151998.

[9] E. Sorokin, "Solid-state materials for few-cycle pulse generation and amplification," in Few-cycle laser pulse generation and its applications. vol. 95, F. X. Kärtner, Ed. Berlin: Springer-Verlag, 2004, pp. 3-71.

[10] L. J. Chen, M. Y. Sander, and F. X. Kärtner, "Kerr-lens mode locking with minimum nonlinearity using gain-matched output couplers," Optics Letters, vol. 35, pp. 2916-2918, Sep 12010.

[11] U. Demirbas, S. Eggert, and A. Leitenstorfer, "Compact and efficient Cr:LiSAF lasers pumped by one single-spatial-mode diode: a minimal cost approach," Journal of Optical Society of America B, vol. 29, pp. 1894-1903, 2012. 